\title{
ESTRATIFICACIÓN POR TAMAÑO DE UNIDADES DE MUESTREO
}

\author{
BASILto A. Rojas \\ Et Colegio de México
}

\section{INTRODUCCIÓN}

EN LA TEORÍ́ Y APLICACIÓN del muestreo el diseño estratificado tiene especial importancia por las múltiples circunstancias prácticas en que se utiliza con grandes ventajas para las estimaciones. Consiste esencialmente en que las unidades de muestreo se reparten en grandes grupos llamados estratos. Para reducir la varianza de la estimación, las unidades dentro de un estrato deberán ser más homogéneas en una o más características pertinentes a la o las estimaciones que se pretenden hacer. Además, se procura que los estratos, homogéneos dentro de sí, difieran lo más posible entre ellos. En esta forma la variabilidad total que posean las unidades de muestreo se puede reducir de manera considerable.

Con el criterio expresado a grandes rasgos arriba se definen los estratos y el problema siguiente es determinar el número de unidades en la muestra correspondiente a cada estrato. Una resolución en este problema es la afijación óptima de Neyman que minimiza la varianza del estimador de la población bajo ciertas restricciones.

El propósito de este estudio es establecer un método analítico para la formación de estratos cuando se conoce el tamaño de todas y cada una de las unidades de muestreo que componen a la población. Por tamaño entendemos el valor $x$ de una característica de la unidad, el que suponemos está altamente correlacionado con la característica $y$ que pretendemos estimar. Suponemos así que se saben las frecuencias absolutas o relativas de $x$ o bien la densidad de probabilidades $f(x)$ en el caso de que $x$ sea continua.

Kendall ${ }^{1}$ examina brevemente la formación de estratos y expone los principios esenciales investigados por Dalenius y Cochran, entre otros. Consideramos que nuestro estudio difiere conceptualmente y además conduce a una metodología más simple.

\section{AFIJACIÓN ÓPTIMA}

El total de unidades de muestreo en la población es $N$. Tenemos $p$ estratos : $i=1, \ldots, p$. El total de unidades en el estrato $i$ es $N_{i}$. Por lo tanto,

1 Maurice G. Kendall y Alan Stuart, The Advanced Theory of Statistics, Vol. 3, Londres, Charles Griffin and Co., 1966. 


$$
N=\sum_{i=1}^{p} N_{i}
$$

En el estrato $i$ se observan $n_{i}$ unidades; seleccionadas al azar de las $N_{i}$. El promedio de la característica $x$ en este estráto es $\bar{x}_{i}$ y el estimador del total de la característica $x$ en la población es $X$ :

$$
X=\sum_{i=1}^{p} N_{i} \bar{x}_{i}
$$

Si $\sigma_{i}^{2}$ es la varianza de la característica $x$ en las unidades del estrato $i$, la varianza de $X$ será: ${ }^{2}$

$$
V(X)=\frac{\sum_{i=1}^{p} N_{i}^{2} \sigma_{i}^{2}}{n_{i}}
$$

Si el costo de investigar $\mathbf{u}$ observar una unidad de muestreo es constante para todos los estratos, la afijación óptima de Neyman nos indica que los $n_{i}$ deberán ser proporcionales a los productos $N_{i} \sigma_{i}$. Si el tamaño total de la muestra es $n$, entonces:

y

$$
n=\sum_{i=1}^{p} n_{i}
$$

$$
n_{i}=n \frac{N_{i} \sigma_{i}}{\Sigma N_{i} \sigma_{i}}
$$

Bien podemos teóricamente concebir un estrato $e$ que tenga un promedio $\bar{x}_{e}$ muy pequeño, un número de unidades $N_{e}$ grande y una $\sigma_{\theta}^{2}$ también grande. Este estrato, aun cuando requiera, de acuerdo con la fórmula (5), un $n_{e}$ relativamente grande, es decir, que esté muy bien representado en la muestra, participaría con muy poco o nada en la estimación de $X$ [fórmula (2)]. Esto nos indica que la afijación de la muestra por el método descrito será defectuosa y sospecharíamos que el calificativo de óptima no es el adecuado.

En diversas investigaciones se ha observado que las varianzas de grupos o estratos son función de los valores medios de los grupos. Si en la estratificación realizada se cumple la ley

$$
\sigma_{i}^{2}=k_{1} \mu_{i}^{2}
$$

en la que $\mu_{i}$ es el valor medio verdadero del estrato $i$ y $k_{1}$ es una constante, los $n_{i}$ serán proporcionales a $N_{i} \mu_{i}$, es decir, a los totales de cada estrato y no se presentaría la dificultad anotada arriba. Pero la suposición (6) no es de ninguna manera una ley general.

2 W. G. Cochran, Sampling Techniques, Nueva York, John Wiley, 1963, 2: ed. 
La fórmula (5) es válida cuando el costo de investigar una unidad de muestreo es el mismo para cualquier estrato. Si las unidades se forman con respecto al tamaño $x$, el costo es independiente del valor de $x$. Si, por ejemplo, la muestra se refiere a producción de trigo de predios agrícolas, el costo sería el mismo para investigar un predio de 100 hectáreas que para el de una hectárea. Efectivamente, el tiempo requerido para hacer la entrevista sería aproximadamente el mismo; sin embargo, la información de, digamos, 200 toneladas de trigo es muy diferente a la de 2 toneladas. Si el costo lo referimos no al de la entrevista por predio, sino a una tonelada de trigo, o en general al valor unitario de $x$, el costo de la entrevista será inversamente proporcional al valor de $x$ que posea la unidad de muestreo. En esta forma el costo medio por unidad en el estrato $i$ sería

$$
C_{i}=\frac{k_{2}}{\mu_{i}}
$$

Bajo esta suposición de costos diferenciales la afijación óptima de $\operatorname{los} n_{i}$ estará dada por

$$
n_{i}=K_{1} N_{i} \sigma_{i} \sqrt{\mu_{i}}
$$

en la que $K_{1}$ es la constante de proporcionalidad, obtenida por la relación (4).

En las distribuciones estadísticas existe una relación, más o menos complicada, entre la desviación estándar $\sigma_{i}$ y la amplitud (recorrido) $\boldsymbol{A}_{\boldsymbol{i}}$ de los datos. Si

$$
\sigma_{i}=k_{3} A_{i}
$$

la expresión (8) quedaría

$$
n_{i}=K_{2} N_{i} A_{i} \sqrt{\mu_{i}}
$$

la cual tendría la ventaja de la simplicidad en la obtención del valor de $A_{i}$ :

$$
A_{i}=X_{i, \text { max }}-X_{i, \min }
$$

o sea la diferencia entre los valores máximos y los mínimos de las observaciones en el estrato $i$.

Puede demostrarse que la expresión (9) es más exacta a medida que el número de estratos es mayor y los $A_{i}$ correspondientes más pequeños. El error $\Delta n_{i}$ en el cálculo del $n_{i}$ debido al error $\Delta \sigma_{i}$ en la determinación de $\sigma_{i}$ es, según la fórmula $(8)$ :

$$
\Delta n_{i}=K_{1} N_{i} \sqrt{\mu_{i}} \Delta \sigma_{i}
$$

si $p$ (número de estratos) es grande y por lo tanto $K$ es prácticamente independiente de $\sigma_{i}$. 
El error relativo será

$$
\frac{\Delta n_{i}}{n_{i}}=\frac{\Delta \sigma_{i}}{\sigma_{i}}
$$

La distribución parcial $f(x)$ de un estrato, en el caso de variable continua, se puede aproximar a una distribución trapecial cuando el número de estratos es grande, que tiene como límites la rectangular, de varianza máxima, y la triangular, de varianza mínima. Por lo tanto, los límites superior e inferior de $\sigma_{i}^{2}$ son como sigue:

$$
\frac{A_{i}^{2}}{18} \leqslant \sigma_{i}^{2} \leqslant \frac{A_{i}^{2}}{12},
$$

y por lo tanto,

$$
0.236 \leqslant \frac{\sigma_{i}}{A_{i}} \leqslant 0.290
$$

El resultado anterior nos señala que el error relativo tendrá como límites

$$
0 \leqslant\left|\frac{\Delta n_{i}}{n_{i}}\right| \leqslant \frac{0.054}{0.236}=0.228
$$

En realidad el error relativo máximo de 0.228 muy difícilmente ocurrirá, pues la constante $k_{3}$ tomará un valor intermedio entre 0.236 y 0.290. Además, dicho error no será sistemático para los estratos. Por otra parte, si tenemos un número grande $p$ de estratos los $n_{i}$ serán relativamente pequeños y los errores en estos $n_{i}$, máximo de $22.8 \%$, tendrán escasa trascendencia. Pero esencialmente la exactitud en los valores de $n_{i}$ es de validez dudosa ya que la estratificación con base en el tamaño $x$ no será la óptima para la variable $y$ que pretendemos estimar y que suponemos tiene alta correlación con la $x$, sin esperar que esa correlación sea uno.

Formación de estratos. Densidad continua

Sea la variable $x$ tal que $x_{\min } \leqslant x \leqslant A+x_{\min }$ y cuya densidad de probabilidades $f(x)$ es también continua y conocida. Subdividamos la población en $p$ estratos, $i=1,2, \ldots, p$, en tal forma que la amplitud (range) de cada estrato sea constante e igual a $A_{e}=A / p$. Los límites inferiores $b_{i-1}$ y superior $b_{i}$ en la variable $x$ del estrato $i$ se obtienen de la fórmula

$$
b_{i}=i \cdot A_{e}+x_{\min }
$$

La media aproximada del estrato $i$ es

$$
\widetilde{\mu_{i}}=\frac{2 i-1}{2} A_{e}+x_{\min }
$$


El número $N_{i}$ de unidades en el estrato $i$ es proporcional a

$$
N_{i} \propto \int_{b_{i-1}}^{b_{i}} f(x) d x
$$

Aplicando estos resultados en la fórmula (10), encontramos que el tamaño óptimo de la muestra $n_{\boldsymbol{i}}$ correspondiente al estrato $i$ es proporcional a

$$
n_{i} \propto A_{e} \sqrt{\frac{2 i-1}{2} \cdot A_{e}+x_{\min }} \int_{b_{i-1}}^{b_{i}} f(x) d x
$$

la que puede expresarse como sigue:

$\mathrm{Si}$

$$
n_{i} \propto \sqrt{\frac{2 i-1}{2}+\frac{x_{\min }}{A_{e}}} \int_{b_{i-1}}^{b_{i}} f(x) d x
$$

$$
K_{i}=\sqrt{\frac{2 i-1}{2}+\frac{x_{\min }}{A_{e}}}, \quad I_{i}=\int_{b_{i-1}}^{b_{i}} f(x) d x
$$

y $n$ es el tamaño total de la muestra, tenemos:

$$
n_{i}=n \frac{K_{i} I_{i}}{\Sigma K_{i} I_{i}}
$$

Si el valor mínimo $x_{\min }$ de la variable $x$ en la población es cero, $K_{i}$ se reduce a

$$
K_{i}=\sqrt{\frac{2 i-1}{2}}
$$

La fórmula (15) es válida cuando el radical es positivo. Para generalizar el procedimiento a una variable $x$ que puede tomar valores negativos se dividiría la población en dos grandes subpoblaciones, una conteniendo los valores positivos de $x$ y la otra los negativos. El proceso indicado sería tomar para cada una de las subpoblaciones los valores absolutos de $x$.

IlUSTRACIONES DEL MÉTODo. Vartable CONTINUA

Presentamos dos ejemplos. El primero se refiere a una distribución rectangular y el segundo a una de tipo hiperbólico que sigue la ley 
de Pareto sobre ingresos familiares. Para simplificar los cálculos se ha considerado un número reducido de estratos, ya que nuestro propósito es explicar el método.

1. Distribución rectangular. Sea la variable $x$ tal que $0<x<1$, y $f(x)=1$. Suponemos $p=4$ estratos. Consideramos $n=2$ y la amplitud de cada estrato $A_{e}=1 / 4=0.25$. Aquí el valor mínimo de $x$ es cero.

Tenemos

$$
\begin{aligned}
& \left.I_{i}=\int_{b_{i-1}}^{b_{i}} f(x) d x=\int_{b_{i-1}}^{b_{i}} 1 \cdot d x=x\right]_{b_{i-1}}^{b_{i}}=b_{i}-b_{i-1}=A_{e}=0.25 \\
& K_{i}=\sqrt{\frac{2 i-1}{2}}
\end{aligned}
$$

Los cálculos aparecen en el cuadro 1.

\section{Cuadro 1}

Formación De estratos y afiJación. Distribución Rectangular. $n=25$

\begin{tabular}{clcccc}
\hline Estrato & Valores de $x$ & $I_{\boldsymbol{i}}$ & $K_{\boldsymbol{i}}$ & $K_{i} I_{\boldsymbol{i}}$ & $n_{\boldsymbol{i}}$ \\
\hline 1 & $0 \quad<x<0.25$ & $\mathbf{0 . 2 5}$ & $\mathbf{0 . 7 1}$ & 0.1775 & 3 \\
2 & $0.25<x<0.50$ & 0.25 & 1.23 & 0.3075 & 6 \\
3 & $0.50<x<0.75$ & 0.25 & 1.58 & 0.3950 & 7 \\
4 & $0.75<x<1.00$ & 0.25 & 1.87 & 0.4675 & 8 \\
\hline Sumas & & & & 1.3475 & 24 \\
\hline
\end{tabular}

Para este caso la afijación óptima simple, con la fórmula (5) da un valor $n_{i}$ constante para todos los estratos, igual a $24 / 4=6$.

2. Distribución de Pareto. Ésta tiene la forma $f(x)=\alpha x^{\beta}$, en la que $x$ es el ingreso por familia, $\alpha$ y $\beta$ son los parámetros de la distribución; $\beta$ es propiamente el parámetro que caracteriza la forma de la distribución y $\alpha$ el de escala, obtenido éste por el requisito de que la integral de $f(x)$ sobre el aspecto de la variable $x$ sea igual a uno. Para el cálculo de los $n_{i}$, fórmula (15), la constante $\alpha$ no es necesaria.

Una estimación tosca del parámetro $\beta$ para la distribución del ingreso familiar mensual en la República Mexicana para 1963 es $\widehat{\beta}=-1.062$. Si $y$ es el número en miles de familias que poseen el ingreso mensual $x$, hemos estimado

$$
y=1.44 \times 10^{6} \cdot x^{-1.062} ; \quad 1000<x<10000 .
$$


El ajuste lo suponemos válido para ingresos mensuales $x$ comprendidos entre $\$ 1000$ y $\$ 10000$.

Nos proponemos estimar una característica altamente correlacionada con el ingreso como pueden ser los gastos familiares. Para ello usaremos como variable auxiliar para la formación de estratos a la $x$ como el ingreso familiar y supondremos $p$ igual a 3 estratos. En este caso, $A=10000-1000=9000 ; A_{e}=9000 / 3=3000$. Así también:

$$
\left.I_{i}=\int_{b_{i-1}}^{b_{i}} x-1.062 d x=-\frac{x^{-0.062}}{0.062}\right]_{b_{i-1}}^{b_{i}}=\frac{b_{i-1}^{-0.062}-b_{i}^{-0.062}}{0.062}
$$

Los valores de $b_{i}$ son $1000,4000,7000$ y $10000 . \quad x_{\min }=1000$. Los resultados de los cálculos se presentan en el cuadro 2 .

\section{Cuadro 2}

Formactón de estratos y afiJactón. Distribución de Pareto

\begin{tabular}{cccccccccc}
\hline $\begin{array}{c}\text { Estrato } \\
i=\end{array}$ & $\begin{array}{c}\text { Valores de } \\
x^{\prime}=x \text { en mi- } \\
\text { les de pesos }\end{array}$ & $\frac{2 i-1}{2} \frac{2 i-1}{2}+\frac{x_{\min }}{A_{e}} K_{i}$ & $I_{i}$ & $K_{i} I_{i}$ & $n_{i}$ & $n_{i}$ \\
\hline 1 & $1<x^{\prime}<4$ & 0.50 & 0.83 & 0.91 & 0.87 & 0.84 & 515 & 622 \\
2 & $4<x^{\prime}<7$ & 1.50 & 1.83 & 1.35 & 0.33 & 0.45 & 275 & 236 \\
3 & $7<x^{\prime}<10$ & 2.50 & 2.83 & 1.68 & 0.20 & 0.34 & 210 & 142 \\
\hline Sumas & & & & & 1.40 & 1.63 & 1000 & 1000 \\
\hline
\end{tabular}

En la última columna del cuadro 2 hemos insertado los valores de la afijación óptima común, que hemos denominado $n_{i}^{\prime}$ :

$$
n_{i}^{\prime} \propto I_{i}
$$

Repetimos que el número de estratos utilizado en el ejemplo es pequeño por facilidad en la presentación y que las fórmulas empleadas son más aproximadas cuando $p$ es grande.

Si la característica $y$ que pretendemos estimar tiene el promedio $\bar{y}_{i}$ en el estrato $i$, el estimador de la media general $\bar{y}$ es

$$
\vec{y}=\frac{\sum I_{i} \bar{y}_{i}}{\sum I_{i}}
$$

cualquiera que haya sido el método empleado para determinar el tamaño de la muestra en los estratos. 


\section{Formación dE los estratos. Densidad discreta}

Éste es el caso común. Tenemos a las unidades de población clasificadas, según la variable $x$, en intervalos de clase y se conoce la frecuencia relativa $f(x)$ correspondiente a cada intervalo. Si éstos tienen la misma amplitud (range) $A_{e}$, podemos utilizar como estratos a estos intervalos y hacer uso de la fórmula (15), en la que $I_{i}=f_{i}$, en donde $f_{i}$ es la frecuencia relativa del intervalo (estrato) $i$. Si los intervalos $A_{i}$ son de tamaño diferente empleamos la fórmula (10) para determinar los $n_{i}$. En esta fórmula (10) $N_{i}$ es la frecuencia absoluta y $\mu_{i}$ es el valor medio del intervalo de clase. Bien puede ser que los estratos agrupen diversos intervalos de clase vecinos; el tratamiento sería el mismo.

Si tenemos conocimiento de las frecuencias absolutas o relativas para cada uno de los intervalos de clase que suponemos de igual amplitud $A_{e}$, nos podemos preguntar qué se gana o se pierde si formamos los estratos con los intervalos de clase originales, en comparación con estratos integrados por dos o más intervalos adyacentes. Sea el intervalo $i$ con frecuencia absoluta $N_{i}$ y el intervalo de clase siguiente $i+1$ con frecuencia absoluta $N_{i+1}$, ambos con la misma amplitud $A_{e}$. Llamemos $R$ a la eficiencia relativa de los dos estratos, con respecto al estrato compuesto. Se puede demostrar que, aproximadamente

$$
R=1+\frac{12 c}{(1+c)^{2}}
$$

en la que $c$ es el cociente de las frecuencias: $c=N_{i} / N_{i+1}$.

Se puede ver que $R$ es mayor que uno y sólo es uno cuando $c$ es cero o infinito. El valor máximo de $R$ es 4 y sucede cuando $c=1$, o sea, $N_{i}=N_{i+1}$, es decir, la subdivisión en estratos es más valiosa cuando las frecuencias de los intervalos vecinos son aproximadamente las mismas. Para $R=1.20$ se requiere un valor de $c$ cercano a 60 .

El resultado anterior nos lleva a la conclusión de que el número de estratos debe ser el máximo posible. Para la estimación del error dentro de cada estrato se exige un mínimo de dos unidades en la muestra, y para este caso el máximo de estratos sería $p=n / 2$. En algunos diseños se hace $p=n$, es decir, se toma una muestra de una unidad por estrato, lo cual maximiza $R$ y una sobrestimación del error de muestreo se obtiene componiendo estratos vecinos. ${ }^{3}$

FORMACIÓN DE ESTRATOS. UNIDADES ORDENADAS POR TAMAÑO

En diversas ocasiones se dispone de la ordenación de las unidades que componen a la población en forma creciente o decreciente con respecto a una variable $x$ que tomamos como tamaño característico de la unidad. También podemos tener el tamaño de cada unidad en tarjetas y es fácil hacer su ordenación. Teniendo esta secuencia de unidades la formación de estratos es fácil siguiendo el procedimiento que describimos a continuación.

s M. H. Hansen, W. N. Hurwitz y W. G. Madow, Sample Survey Methods and Theory, Vol. II, Nueva York, John Wiley, 1953. 
Supongamos tener $p$ estratos y que en el estrato $i$ hemos obtenido una muestra al azar de $n_{i}$ unidades. La varianza de $X$, el total estimado de la población, será

Si ahora

$$
V(X)=\Sigma N_{i}^{2} \frac{\sigma_{i}^{2}}{n_{i}}, i=1,2, \ldots, p
$$

tendremos

$$
\sigma_{i}^{2} \leqslant G
$$

$$
V(X) \leqslant G \Sigma \frac{N_{i}^{2}}{n_{i}}
$$

Por otra parte, si hiciéramos una afijación proporcional, es decir,

y sustituyendo

$$
n_{i}=\frac{N_{i}}{N} n
$$

$$
V(X) \leqslant \frac{G N^{2}}{n}
$$

$y$, si $\bar{x}$ es la estimación de la media de la población,

$$
V(\bar{x})<\frac{G}{n}
$$

Si la amplitud total (recorrido)) de la población es $A$ y esto se divide en $p$ intervalos iguales, correspondiendo cada uno de ellos a un estrato, el recorrido de cada estrato será el mismo e igual a $A_{e}=A / p$. La varianza máxima con un intervalo $\mathrm{A}_{e}$ es $A_{e}^{2} / 2$ y tomaría este valor cuando el estrato tuviera dos unidades y con valores en los extremos del intervalo. Por lo tanto,

$$
V(\bar{x})<\frac{1}{2 n}\left(\frac{A}{p}\right)^{2}
$$

Pero si suponemos que el tamaño de muestra para cada estrato es cuando menos igual a $2, n>2 p$, y así podemos escribir,

de donde,

$$
V(\bar{x})<\frac{A^{2}}{4 p^{3}},
$$

$$
p<\left(\frac{A^{2}}{4 V(\bar{x})}\right)^{1 / 3}=\left(\frac{\frac{A}{\mu}}{2 \frac{\sigma_{\bar{x}}}{\mu}}\right)^{2 / 3}
$$

En la última expresión $\mu$ es la media de la población, $\sigma_{\bar{x}} / \mu$ es el coefi- 
ciente de variación de la media de la muestra y su valor mide el grado de aproximación que nos proponemos alcanzar.

La expresión (18) proporciona el límite superior en el número de estratos en que dividimos la población. Consideramos que en muchas situaciones prácticas el valor máximo de la varianza de un estrato es inferior al señalado de $A_{e}^{2} / 2$, porque habrá un número $N_{i}$ relativamente grande y con valores de $x$ distribuidos dentro del intervalo correspondiente. Por ello recomendamos el uso de la siguiente expresión (19) para determinar el número de estratos,

$$
p=\left(\frac{\frac{A}{\mu}}{3 \frac{\sigma_{\bar{x}}}{\mu}}\right)^{2 / 3}
$$

Obtenido el valor de $p$ con la fórmula anterior, determinamos $A_{e}=A / p$. El estrato $i$ estará formado por las unidades que posean valores $x$ comprendidos en el intervalo

$$
x_{\max }-(i-1) A_{e}<x<x_{\text {max }}-i \cdot A_{e}
$$

$x_{\max }$ es el valor máximo de $x$ que posean una o más unidades en la población.

Una vez determinados los estratos, conocemos los $N_{i}$. Para un tamaño total $n$, podemos afijar $\operatorname{los} n_{i}$ proporcionalmente a $\operatorname{los} N_{i}$, o bien de acuerdo con la fórmula,

$$
n_{i} \propto N_{i} \sqrt{\mu_{i}}
$$

en la que $\mu_{i}$ es la media del estrato $i$. Esta última fórmula para $n_{i}$ es la correspondiente a la expresión (10) suponiendo que los $A_{i}$ son constantes e iguales a $A_{e}$. En esta afijación $n_{i}$ será siempre mayor o igual a 2.

En toda nuestra discusión no hemos considerado el ajuste en el tamaño de la muestra por finitud de la población. Si esto se presenta se haría el ajuste conocido.

No debe extrañarnos que después de construir los estratos por el procedimiento descrito lleguemos a un número de ellos distinto de $p$. La diferencia será despreciable para nuestro propósito.

\section{RESUMEN}

En el estudio se desarrollan conceptos teóricos que determinan la influencia de la formación de estratos en la aproximación de los estimados de una población. Se examina primeramente el caso de una población que sigue una función de probabilidades continua y en seguida el caso de una función discreta. En el último caso se señala el criterio de formación de estratos cuando las unidades que compo- 
nen a la población estén ordenadas conforme a la magnitud de la variable $x$, definido como tamaño de la unidad.

Determinados los estratos, el aspecto siguiente es determinar el tamaño de muestra en cada estrato. En este estudio se introduce el criterio de considerar como costo de investigar a la unidad $i$ un valor inversamente proporcional a la magnitud $x_{i}$ de su tamaño correspondiente. Se señalan las razones para proceder en esta forma. 